\title{
ON THE COMBINATORIAL SCHOENFLIES CONJECTURE
}

\author{
ERHARD LUFT
}

Introduction. The combinatorial version of the Schoenflies conjecture in dimension $n$ states: A combinatorial $(n-1)$-sphere on a combinatorial $n$-sphere decomposes the latter into two combinatorial $n$ cells. The cases $n=1,2$ are obvious, the case $n=3$ was solved by J. W. Alexander [1], see also W. Graeub [5], and E. Moise [8]. Nothing is known for $n>3$ (compare J. F. Hudson and E. C. Zeeman in $[6$, p. 729]). On the other hand the following form of the Hauptvermutung for spheres is proved: If a combinatorial $n$-dimensional manifold is homeomorphic an $n$-dimensional sphere, then it is a combinatorial $n$-sphere if $n \neq 4$. This was proved by S. Smale for $n \neq 4,5,7$ in [9], and improved by E. C. Zeeman to $n \neq 4$ (unpublished). I would like to thank Professor Zeeman for pointing out this result to me. Further a generalized Schoenflies theorem was proved by $\mathrm{M}$. Brown in [3]. If this Hauptvermutung were true for all $n$, a simple induction on the dimension $n$ would prove the combinatorial Schoenflies conjecture for all $n$ (compare Theorem 6 ). In the following we show that either the combinatorial Schoenflies conjecture is true for all dimensions $n$, or it is false for all dimensions $n>3$.

Notation. We are dealing with finite simplicial complexes, hereafter called complexes. An $n$-complex is a complex such that each simplex is a face of an $n$-dimensional simplex of the complex. If $K, L$ are complexes then $K \cup L, K \cap L, K \cdot L$ denote the union, intersection, and the simplicial product of the complexes $K$ and $L$. A combinatorial $n$-cell is a complex which has a subdivision isomorphic a subdivision of an $n$-simplex. And a combinatorial $n$-sphere is a complex which has a subdivision isomorphic to a subdivision of the boundary of an $(n+1)$-simplex. The link $L(s, K)$ of the simplex $s$ of the complex $K$ is the subcomplex consisting of those simplexes $s^{\prime}$ of $K$ with $s$ and $s^{\prime}$ are faces of a simplex in $K$ and $s^{\prime}$ has no vertices in common with $s$. An $n$-dimensional combinatorial manifold is an $n$-complex such that the link of each vertex is a combinatorial $(n-1)$-sphere or a combinatorial $(n-1)$-cell. It is called closed, if its boundary is empty.

Theorem 1 (J. W. Alexander [2]). Let $S^{n}$ and $S^{k}$ be combinatorial spheres of dimensions $n$ and $k$, then the product $S^{n} \cdot S^{k}$ is a combinatorial $(n+k+1)$-sphere. Let $S^{n}$ be a combinatorial $n$-sphere and $E^{k}$ a com-

Received by the editors August 28, 1964 
binatorial $k$-cell, then the product $S^{n} \cdot E^{k}$ is a combinatorial $(n+k+1)$ cell.

Theorem 2 (J. W. Alexander [2]). Let $S^{n}$ be a combinatorial $n$ sphere with the decomposition $S^{n}=E_{1}^{n} \cup E_{2}^{n}$, where $E_{1}^{n}$ and $E_{2}^{n}$ are $n$ complexes such that $E_{1}^{n} \cap E_{2}^{n}=S^{n-1}$ is a combinatorial $(n-1)$-sphere. If $E_{1}^{n}$ is a combinatorial $n$-cell, then $E_{2}^{n}$ is also a combinatorial $n$-cell.

TheOREM 3 (H. KNESER [7]). Let $M^{n}, n \geqq 1$, be a closed connected n-dimensional combinatorial manifold. Suppose $H_{1}\left(M^{n} ; Z_{2}\right)=0$ if $n \geqq 2$, where $H_{1}\left(M^{n} ; Z_{2}\right)$ is the first homology group of $M^{n}$ with coefficients in $Z_{2}$. Then any closed connected $(n-1)$-dimensional combinatorial manifold $M^{n-1}\left(M^{0}=S^{0}\right)$ on $M^{n}$ determines a unique decomposition of $M^{n}$ into two $n$-complexes $K_{1}$ and $K_{2}$ with $M^{n}=K_{1} \cup K_{2}$ and $K_{1} \cap K_{2}$ $=M^{n-1}$.

TheOREM 4. The combinatorial Schoenflies conjecture is true in dimension $n$ if and only if a combinatorial $n$-sphere $S^{n}$ on a combinatorial $(n+1)$-sphere $S^{n+1}$ decomposes the latter into two combinatorial manifolds $E_{1}^{n+1}$ and $E_{2}^{n+1}$ with $E_{1}^{n+1} \cup E_{2}^{n+1}=S^{n+1}$ and $E_{1}^{n+1} \cap E_{2}^{n+1}=S^{n}$.

Proof. The "if" part: Let $S^{n-1}$ be a combinatorial $(n-1)$-sphere on the combinatorial $n$-sphere $S^{n}$. From Theorem 3 we have the decomposition of $S^{n}$ into two $n$-complexes $E_{1}^{n}$ and $E_{2}^{n}$ with $E_{1}^{n} \cup E_{2}^{n}=S^{n}$ and $E_{1}^{n} \cap E_{2}^{n}=S^{n-1}$. Let $S^{0}=\{x, y\}$ be a 0 -sphere. We form the simplicial product $S^{n} \cdot S^{0}=E_{1}^{n} \cdot S^{0} \cup E_{2}^{n} \cdot S^{0}$. Of course $E_{1}^{n} \cdot S^{0} \cap E_{2}^{n} \cdot S^{0}$ $=S^{n-1} \cdot S^{0}$. But $S^{n-1} \cdot S^{0}$ is now a combinatorial $n$-sphere on the combinatorial $(n+1)$-sphere $S^{n} \cdot S^{0}$. And $E_{1}^{n} \cdot S^{0}$ and $E_{2}^{n} \cdot S^{0}$ are then by hypothesis combinatorial manifolds. We compute the following links: $L\left(x, E_{1}^{n} \cdot S^{0}\right)=E_{1}^{n}$ and $L\left(x, E_{2}^{n} \cdot S^{0}\right)=E_{2}^{n} . E_{1}^{n}$ and $E_{2}^{n}$ must be $n$-cells.

The "only if" part: From Theorem 3 we have that $S^{n+1}$ is decomposed into two $(n+1)$-complexes $E_{1}^{n+1}$ and $E_{2}^{n+1}$ with $S^{n+1}$ $=E_{1}^{n+1} \cup E_{2}^{n+1}$ and $E_{1}^{n+1} \cap E_{2}^{n+1}=S^{n}$. To show that these $(n+1)$ complexes are combinatorial manifolds, we have only to check that the links of their vertices are either combinatorial $n$-spheres or combinatorial $n$-cells. For a vertex $e$ not in $S^{n}$ we have $L\left(e, E_{i}^{n+1}\right)$ $=L\left(e, S^{n+1}\right), i=1,2$, and this link is therefore a combinatorial $n$ sphere. If the vertex $e$ lies on $S^{n}$ then the combinatorial $(n-1)$-sphere $L\left(e, S^{n}\right)$ decomposes the combinatorial $n$-sphere $L\left(e, S^{n+1}\right)$ by hypothesis into two combinatorial $n$-cells, which lie in $E_{1}^{n+1}$ and $E_{2}^{n+1}$. These $n$-cells are the links of $e$ in $E_{1}^{n+1}$ and $E_{2}^{n+1}$.

Corollary 1. If the combinatorial Schoenflies conjecture is true in dimension $n$, then it is also true in dimension $n-1$, and therefore in all dimensions up to $n$. 
Theorem 5 (M. Brown [3] AND [4]). Let $S^{n}$ be a combinatorial $n$-sphere and $S^{n-1}$ a combinatorial $(n-1)$-sphere on $S^{n}$. Then $S^{n-1} d e-$ composes $S^{n}$ into two complexes $E_{1}^{n}$ and $E_{2}^{n}$ with $S^{n}=E_{1}^{n} \cup E_{2}^{n}, E_{1}^{n} \cap E_{2}^{n}$ $=S^{n-1}$, and $E_{1}^{n}, E_{2}^{n}$ are topological $n$-cells, i.e. $E_{1}^{n}$ and $E_{2}^{n}$ are homeomorphic to an $n$-simplex.

Proof. $S^{n-1}$ is "bi-collared" in $S^{n}$, and the generalized Schoenflies theorem of [3] can be applied (see [4]). By the combinatorial Hauptvermutung for $n$-spheres we mean the statement, that if an $n$-dimensional combinatorial manifold is homeomorphic to an $n$-sphere then it is a combinatorial $n$-sphere.

THEOREM 6. If the combinatorial Schoenflies conjecture is true in dimension $n-1$, and if the combinatorial Hauptvermutung for $n$-spheres is true, then the combinatorial Schoenflies conjecture is true in dimension $n$.

Proof. Suppose $S^{n-1}$ is a combinatorial $(n-1)$-sphere on the combinatorial $n$-sphere $S^{n}$. From Theorem 4 we have the decomposition $S^{n}=E_{1}^{n} \cup E_{2}^{n}$, where $E_{1}^{n}$ and $E_{2}^{n}$ are $n$-dimensional combinatorial manifolds, and $E_{1}^{n} \cap E_{2}^{n}=S^{n-1}$. By Theorem 5 we know that $E_{1}^{n}$ and $E_{2}^{n}$ are topological $n$-cells. We consider the $n$-dimensional combinatorial manifold $\widetilde{S}^{n}=E_{1}^{n} \cup S^{n-1} \cdot E^{0}$, where $E^{0}=\{x\}$ is a 0 -cell. Since $E_{1}^{n}$ and $S^{n-1} \cdot E^{0}$ are topological $n$-cells, $\widetilde{S}^{n}$ is homeomorphic to the $n$-sphere, and by hypothesis $\widetilde{S}^{n}$ is a combinatorial $n$-sphere. Since $S_{1}^{n-1} \cdot E^{0}$ is a combinatorial $n$-cell, we can apply Theorem 2 and conclude that $E_{1}^{n}$ is also a combinatorial $n$-cell. And by the same argument $E_{2}^{n}$ is a combinatorial $n$-cell too.

Theorem 7 (S. Smale [9] And E. C. Zeeman). The combinatorial Hauptvermutung for $n$-spheres is true for $n \neq 4$.

COROLlaRy 2. Either the combinatorial Schoenflies conjecture is true for all dimensions $n$, or it is false for all dimensions $n>3$.

Corollary 3. If the combinatorial Schoenfies conjecture can be proved for one dimension $n_{0}>3$, then it is true for all dimensions $n$. If a counterexample to the combinatorial Schoenflies conjecture can be found in one dimension $n_{0}>3$, then the conjecture is false for all dimensions $n>3$, and subsequently the combinatorial Hauptvermutung for 4spheres would be false.

\section{REFERENCES}

1. J. W. Alexander, On the subdivision of 3-space by a polyhedron, Proc. Nat. Acad. Sci. U.S.A. 10 (1924), 6-8. 
2. - The combinatorial theory of complexes, Ann. of Math. 31 (1930), 292320.

3. M. Brown, $A$ proof of the generalized Schoenflies theorem, Bull. Amer. Math. Soc. 66 (1960), 74-76.

4. - Locally flat embeddings of topological manifolds, Topology of 3-manifolds, pp. 83-91, Prentice-Hall, New York, 1962.

5. W. Graeub, Die semilinearen Abbildungen, S.-B. Heidelberger Akad. Wiss. Math.-Natur. Kl. (1950), 205-272.

6. J. F. Hudson and E. C. Zeeman, On regular neighborhoods, Proc. London Math. Soc. (3) 14 (1964), 719-745.

7. H. Kneser, Ein topologischer Zerlegungssatz, Proc. Roy. Acad. Amsterdam 27 (1924), 601-616.

8. E. Moise, Affine structures in 3-manifolds. II. Positional properties of 2-spheres, Ann. of Math. 55 (1953), 172-176.

9. S. Smale, Differentiable and combinatorial structures on manifolds, Ann. of Math. 74 (1961), 498-502.

University of British Columbia, Vancouver, B. C., Canada 